\title{
The Infrared Surface Brightness Fluctuation Distances to the Hydra and Coma Clusters
}

\author{
Joseph B. Jensen ${ }^{1}$ \\ John L. Tonry \\ and \\ Gerard A. Luppino \\ Institute for Astronomy, University of Hawaii \\ 2680 Woodlawn Drive, Honolulu, HI 96822 \\ e-mail: jjensen@gemini.edu, jt@avidya.ifa.hawaii.edu, ger@hokupa.ifa.hawaii.edu
}

\begin{abstract}
We present IR surface brightness fluctuation (SBF) distance measurements to NGC 4889 in the Coma cluster and to NGC 3309 and NGC 3311 in the Hydra cluster. We explicitly corrected for the contributions to the fluctuations from globular clusters, background galaxies, and residual background variance. We measured a distance of $85 \pm 10 \mathrm{Mpc}$ to NGC 4889 and a distance of $46 \pm 5 \mathrm{Mpc}$ to the Hydra cluster. Adopting recession velocities of $7186 \pm 428 \mathrm{~km} \mathrm{~s}^{-1}$ for Coma and $4054 \pm 296 \mathrm{~km} \mathrm{~s}^{-1}$ for Hydra gives a mean Hubble constant of $H_{0}=87 \pm 11 \mathrm{~km} \mathrm{~s}^{-1} \mathrm{Mpc}^{-1}$. Corrections for residual variances were a significant fraction of the SBF signal measured, and, if underestimated, would bias our measurement towards smaller distances and larger values of $H_{0}$. Both NICMOS on the Hubble Space Telescope and large-aperture ground-based telescopes with new IR detectors will make accurate SBF distance measurements possible to $100 \mathrm{Mpc}$ and beyond.
\end{abstract}

Subject headings: distance scale — galaxies: clusters: individual (Hydra, Coma) galaxies: individual (NGC 3309, NGC 3311, NGC 4889) — galaxies: distances and redshifts

\section{Introduction}

Measuring accurate and reliable distances is a critical part of the quest to measure the Hubble constant $H_{0}$. Until recently, different techniques for estimating extragalactic distances

\footnotetext{
${ }^{1}$ Currently with the Gemini 8-m Telescopes Project, 180 Kinoole St. Suite 207, Hilo, HI 96720. The Gemini 8-m Telescopes Project is managed by the Association of Universities for Research in Astronomy, for the National Science Foundation and the Gemini Board, under an international partnership agreement.
} 
produced results that differed by as much as a factor of two, while the statistical uncertainties of individual techniques were much smaller. Identifying and removing systematic errors from distance measurements has been a difficult and lengthy process, but progress is now being made on a number of fronts towards resolving discrepancies in distance measurements. Detailed summaries of many different distance measurement techniques are found in the reviews by Jacoby et al. (1992) and in the proceedings of a recent symposium on the extragalactic distance scale (Livio, Donahue, \& Panagia 1997).

Reliably determining $H_{0}$ also requires the measurement of accurate radial velocities. In the local universe, peculiar velocities are larger than the Hubble flow we must measure. To avoid this problem, we must accurately measure distances great enough that local velocity perturbations are negligible. Recently, however, several studies have measured residual velocities on uncomfortably large scales (Lynden-Bell et al. 1988; Mould et al. 1991; Mathewson, Ford, \& Buchhorn 1992; Lauer \& Postman 1994) Large-scale motions are difficult to explain with standard models of hierarchical structure formation, so it is crucial to our understanding of cosmology that we confirm the reality of such large flows with accurate distance measurements. Large-scale motions can also bias measurements of $H_{0}$ so it is important that accurate distances be measured to galaxies in many directions and to distances beyond $10,000 \mathrm{~km} \mathrm{~s}^{-1}$.

Surface brightness fluctuations (SBFs) have proven to be a remarkably accurate distance indicator (Tonry et al. 1997). Ground-based optical SBFs reach $\sim 3000 \mathrm{~km} \mathrm{~s}^{-1}$; IR SBFs are some 30 times brighter than optical fluctuations, and can potentially reach much greater distances. We explored the advantages of measuring SBFs in the $K^{\prime}$ band $(2.1 \mu \mathrm{m})$ and calibrated the $K^{\prime}$ SBF distance scale (Jensen, Tonry, \& Luppino 1998, hereafter JTL; Jensen, Luppino, \& Tonry 1996). From these studies we learned that it is important to account for the contributions to the IR SBFs from globular clusters and background galaxies. JTL and Jensen et al. (1996) also concluded that residual noise in IR images that is correlated from pixel to pixel can dominate SBFs when the ratio of signal to noise $(S / N)$ is small.

This paper presents measurements of the $K^{\prime}$ SBF distances to NGC 3309 and NGC 3311 in the Hydra cluster and to NGC 4889 in the Coma cluster to establish IR SBF as a technique useful to $\sim 7000 \mathrm{~km} \mathrm{~s}^{-1}$. This is the first step towards an accurate measurement of the IR SBF Hubble constant and a better determination of peculiar velocities on scales of $\sim 5000 \mathrm{~km} \mathrm{~s}^{-1}$. At $7000 \mathrm{~km} \mathrm{~s}^{-1}$, the peculiar velocity of the Coma cluster should be less than $10 \%$ of its Hubble velocity. At half the distance to Coma, SBF measurements of Hydra cluster galaxies can be used to test our techniques and to confirm the reliability of the more distant Coma measurements. Tully-Fisher and $D_{n}-\sigma$ distance measurements both show Hydra to be nearly at rest with respect to the Hubble flow (Han \& Mould 1992; Faber et al. 1989), so we can use the distance to Hydra to confirm the Coma cluster determination of $H_{0}$. 


\section{Observations and Data Reduction}

The data described in this paper were obtained using the University of Hawaii $2.24 \mathrm{~m}$ telescope on 1996 March 1 and April 25-28. We used the new science-grade "HAWAII" infrared array, a $1024^{2}$-pixel HgCdTe detector mounted in the QUIRC camera (Hodapp et al. 1996). At f/10, QUIRC has a pixel scale of $0{ }^{\prime} 1886$ pixel $^{-1}$, producing a field of view $3 ! 2$ on a side. We observed using the University of Hawaii $K^{\prime}$ filter centered at $2.1 \mu \mathrm{m}$, which is much less sensitive to the thermal background than the standard $K$ filter (Wainscoat \& Cowie 1992). Conditions on these nights were photometric and the seeing at $K^{\prime}$ averaged 0.75 FWHM. The dark current of the array was less than $1 \mathrm{e}^{-} \mathrm{s}^{-1}$ and the correlated double-sample read noise was $\lesssim 15 \mathrm{e}^{-}$. The banded sensitivity patterns and anomalous dark current features common in earlier IR arrays have been reduced significantly in the new HAWAII device. In addition, the new array has far fewer bad pixels $(\$ 1 \%)$ and higher quantum efficiency than previous IR arrays. The dark current in this device is locally sensitive to bright objects, and residual images of bright or saturated objects persist for several minutes. Individual integration times were kept short enough to prevent the centers of the galaxies from approaching saturation, and sky frames were acquired after every galaxy image.

We integrated for 90 to $120 \mathrm{~s}$ at a time on each galaxy, and then moved $\sim 400^{\prime \prime}$ to sample the sky. Sky fields were chosen to be relatively free of galaxies and bright stars. Exposure times, sky brightnesses, and other observational parameters are listed in Table 1. UKIRT faint standard stars were observed several times during each night (Casali \& Hawarden 1992). Observing conditions were excellent during both 1996 March and April, and our photometric zero point was internally consistent to $0.01 \mathrm{mag}$. We used the same standard stars and filter described in the $K^{\prime}$ SBF calibration study (JTL) to minimize systematic uncertainties. The atmospheric extinction coefficient measured during the 1996 April run was 0.065 mag air mass ${ }^{-1}$ and was consistent enough from night to night to be used for the entire run. During 1996 March the extinction coefficient was determined to be 0.115 mag air mass ${ }^{-1}$. NGC 3309 and NGC 3311 were observed during both runs, and the photometric data listed in Table 1 are weighted mean values.

We adopted a galactic foreground extinction correction of $A_{K^{\prime}}=0.068 A_{B}$ based on the results of Cohen et al. (1981) and as described in JTL. Extinction values in the $B$ band were taken from Burstein \& Heiles (1984). Extinction corrections are small, and their associated uncertainties are negligible. $\mathrm{K}$ corrections were computed for the redshifts of the Coma and Hydra clusters by computing $K$-band fluctuation magnitudes for model stellar populations at redshifts in the range $0.0 \leq z \leq 0.03$. We used the $\mathrm{K}$ corrections listed in Table 6 of JTL, computed using the stellar population models from Worthey (1994) and G. Worthey (1997, private communication) assuming a metallicity of $[\mathrm{Fe} / \mathrm{H}]=0$. For the Coma cluster at $z=0.025$, the K correction is between 0.003 and -0.011 mag for stellar populations with ages from 12 to 17 Gyr. For old, metal-rich stellar populations, the $\mathrm{K}$ correction is negligible.

The first step in the image reduction procedure was to create an average sky image. We 
Table 1. Hydra and Coma Observational Data

\begin{tabular}{|c|c|c|}
\hline & Hydra & Coma \\
\hline Galaxies. & NGC 3309, NGC 3311 & NGC 4889 \\
\hline Observation dates... & $\begin{array}{c}1996 \text { Mar } 1, \\
1996 \text { Apr } 25,26,28\end{array}$ & 1996 Apr $25-28$ \\
\hline$m_{1}(\operatorname{mag})^{\mathrm{a}} \ldots \ldots \ldots \ldots \ldots \ldots \ldots$ & $23.02 \pm 0.01$ & $22.99 \pm 0.01$ \\
\hline $\sec z \ldots \ldots \ldots \ldots \ldots \ldots$ & 1.594 & 1.287 \\
\hline$A_{a t m}(\mathrm{mag} / \sec z) \ldots \ldots \ldots$ & 0.088 & 0.065 \\
\hline Seeing FWHM ......... & 0.85 & 0.75 \\
\hline Sky brightness (mag $\operatorname{arcsec}^{-2}$ ) & 13.62 & 13.70 \\
\hline Sky brightness $\left(\mathrm{e}^{-} \mathrm{s}^{-1}\right.$ pixel $\left.^{-1}\right)$ & 206 & 185 \\
\hline Residual sky $\left(\mathrm{e}^{-} \mathrm{s}^{-1}\right.$ pixel $\left.^{-1}\right)$ & $0.62 \pm 0.10$ & $0.88 \pm 0.05$ \\
\hline$t_{\exp }(\mathrm{s}) \ldots \ldots \ldots \ldots \ldots \ldots \ldots$ & 90 & 120 \\
\hline$t_{t o t}(\mathrm{~s}) \ldots \ldots \ldots$ & 9,090 & 15,960 \\
\hline$A_{B}(\mathrm{mag})^{\mathrm{b}}$. & 0.17 & 0.05 \\
\hline$A_{K^{\prime}}(\mathrm{mag})$. & 0.012 & 0.003 \\
\hline
\end{tabular}

${ }^{\text {a }}$ Magnitude of a source yielding $1 \mathrm{e}^{-} \mathrm{s}^{-1}$.

${ }^{\mathrm{b}}$ Burstein \& Heiles 1984 
masked stars and galaxies in individual sky frames before averaging 5 to 7 images to form a sky image. Individual exposures were $120 \mathrm{~s}$, so a stack of 7 sky frames spans about 30 minutes of observing time, which is roughly the time scale over which variations in sky brightness typically begin to be significant. Next, an average sky image was subtracted from each galaxy frame prior to dividing by a normalized flat field image. Dark current is subtracted along with the sky background. Cosmic rays were then removed from each image. Finally, the cleaned images were registered to the nearest pixel and good pixels averaged to form the final galaxy image. Sub-pixel registration was not used because it modifies the noise characteristics of the background, introducing correlations in the noise between pixels that were uncorrelated in the individual images. The registered $K^{\prime}$ images are displayed in Figures 1 and 2.

Because the sky level changes on short time scales, sky subtraction left a non-zero background level that we measured and removed. We assumed the residual sky offset was constant across the field of view and applied a simple offset correction. The galaxies we observed are large and fill most of the field of view. To determine the residual sky level, we fitted a deVaucouleurs $r^{1 / 4}$ profile to the surface brightness of the galaxy measured in elliptical annuli. We then adjusted the background level to minimize the deviation from an $r^{1 / 4}$ profile. The residual sky corrections listed in Table 1 were applied before proceeding with the SBF analysis.

\section{Surface Brightness Fluctuation Analysis}

The techniques for measuring distances using SBFs were first laid out by Tonry \& Schneider (1988). The procedures used to measure SBFs in this study are the same as those presented in detail by JTL. We summarize only the principal steps here. First we fitted a smooth model to the galaxy surface brightness after the residual sky brightness had been subtracted. Extraneous objects were masked before fitting. After the galaxy had been fitted, subtracted, and compact sources masked, residual large-scale variations were fitted and subtracted. The masked residual image was then Fourier transformed and the two-dimensional power spectrum computed. The power spectrum is the convolution of the stellar SBFs with an expectation power spectrum $E(k)$, which includes the normalized power spectrum of the point spread function (PSF), the galaxy surface brightness profile, and the power spectrum of the mask. The details of the procedure are described by JTL.

The power spectrum was fitted with the sum of the SBF power $P_{0}$ multiplied by the expectation power spectrum $E(k)$ and a white noise component $P_{1}$. We measured $P_{0}$ in annular regions centered on the galaxy. The contribution to the fluctuation power from undetected GCs and background galaxies $\left(P_{r}\right)$ was estimated and subtracted from $P_{0}$. The residual spatial variance $\left(P_{g}\right)$ remaining after subtracting the bright background and galaxy was also estimated and subtracted from $P_{0}$. The methods we used to determine $P_{r}$ and $P_{g}$ are described in detail 
below and by JTL. The fluctuation magnitude is defined as

$$
\bar{m}_{K^{\prime}}=-2.5 \log \left(P_{0}-P_{r}-P_{g}\right)+m_{1}-A_{a t m} \sec z-A_{K^{\prime}}
$$

where $P_{0}$ is the SBF power in $\mathrm{e}^{-} \mathrm{s}^{-1}$ pixel $^{-1}$ and $m_{1}$ is the magnitude of a source yielding one $\mathrm{e}^{-} \mathrm{s}^{-1}$ at zero air mass.

To determine the size of the contribution to the stellar SBFs from undetected GCs and background galaxies $P_{r}$, we first measured the magnitudes of all the objects in the image. GC and galaxy luminosity functions were fitted as a function of radius from the center of the galaxy (as described by JTL). Objects brighter than the completeness limit were masked, and we integrated the luminosity functions beyond the completeness cutoff to compute the numbers of faint unmasked GCs and galaxies that add variance to $P_{0}$. The size of the $P_{r}$ correction is clearly a function of the limiting magnitude of the observation. JTL showed that even though the contrast between stellar SBFs and GCs is a factor of ten higher in the IR than at optical wavelengths, the fraction of the total IR SBF power from GCs and galaxies can still be $\sim 50 \%$, or larger in galaxies like NGC 3311 that have unusually large populations of GCs.

At $K^{\prime}$, the sky background is hundreds of times brighter than in the optical bands. Even when IR integrations are long enough to detect SBFs, the majority of the GCs remain undetected in the background noise. When the fit to the GC and galaxy luminosity functions is derived from only the few brightest objects, it is poorly constrained and the uncertainty in $P_{r}$ is the principal source of uncertainty in $\bar{m}_{K^{\prime}}$. JTL showed that using deep optical images to identify the locations of GCs and background galaxies undetected in the IR can reduce $P_{r}$ to negligible levels. From the optical image, a mask was created and used to remove objects from the $K^{\prime}$ image that are too faint to be detected at $K^{\prime}$. With much lower background levels, the optical image reaches a much fainter limiting magnitude and the resulting mask removes a greater fraction of the GCs and galaxies. We used the composite optical/IR technique to remove the GC and galaxy contributions from the Coma and Hydra data.

For the observations of relatively nearby galaxies studied by JTL, the residual variance $P_{r}$ from GCs and galaxies after applying a mask generated from deep $I$-band images was less than $1 \%$ and was ignored. For the Coma cluster data presented here, the optical images only reach GCs two to three mag brighter than the peak of the luminosity function, requiring a careful correction for the the clusters and galaxies that remain unmasked. For the present study, we developed a straightforward method for estimating $P_{r}$ in the $K^{\prime}$ observations based on the $I$-band photometry. We transformed the $I$-band magnitudes to $K^{\prime}$-band assuming a uniform color for each of the GC and galaxy populations. Values were computed for the $(I-K)$ colors of the GCs and galaxies by adopting mean $(V-K)$ colors from Frogel et al. (1978) and mean $(V-I)$ colors from Ajhar \& Tonry (1994) (GCs) and JTL (galaxies). We adopted $(I-K)=1.22$ for GCs and 2.1 for galaxies. The coordinates of the objects were scaled and translated to the frame defined by the $K^{\prime}$ image. We then fitted the luminosity functions, created a mask of objects brighter than the completeness limit, and estimated the $K$-band correction $P_{r}$ for our observations. 
To fit the GC luminosity function, we assumed the width, form, and peak magnitude of the GCLF, which required a preliminary estimate of the distance. Fitting the galaxy luminosity function required a power-law slope and normalization. JTL described the detailed forms of the luminosity functions used to estimate $P_{r}$. The fluctuation magnitude is not sensitive to the fit parameters provided that objects $\sim 2$ mag from the GCLF peak and brighter are measured, fitted and masked. The variance is dominated by the brightest objects, so $\bar{m}_{K^{\prime}}$ is insensitive to errors in the fit to the faint end of the luminosity functions.

\section{4. $\quad K^{\prime}$ SBF Magnitudes and Distances}

To compute the fluctuation magnitude and distance to each galaxy, we first measured the $K^{\prime}$ SBF power in three annular regions centered on each galaxy. Radii and $P_{0}$ powers measured are listed in Table 2. The power spectra are plotted in Figures 3 and 4 with the individual components $P_{0} \times E(k)$ and $P_{1}$, and their sum. We determined the fluctuation magnitudes $\bar{m}_{K^{\prime}}$ corrected for residual variances using Equation 11. Finally, the absolute fluctuation magnitude $\bar{M}_{K^{\prime}}$ from JTL was used to compute the distance moduli.

\subsection{Residual Variance from Globular Clusters and Galaxies}

JTL described the advantages of using optical images to identify and mask GCs and background galaxies in IR images prior to performing the SBF analysis. We applied this technique (as described in Section 3) to the data presented in this paper. We used the $I$-band image of NGC 4889 kindly provided by J. Blakeslee (1997, private communication) to identify GCs and galaxies down to approximately $K=22.5$. A. Dressler (1997, private communication) generously made a deep $I$-band image of the Hydra galaxies available, allowing us to reach a similar limiting magnitude in our Hydra data.

To constrain the GCLF at $K^{\prime}$, we adopted uniform GC and galaxy colors and translated $I$ magnitudes to $K$ as described in the previous section. We then performed the fit to the GC and galaxy luminosity functions as if the data had been collected from a $K$-band image and created a mask of all objects brighter than the completeness limit. The luminosity functions were integrated beyond the cutoff magnitude as a function of radius from the center of each galaxy, and we computed the residual variance $P_{r}$ for each annular region. $P_{r}$ values are listed in Table 2. The luminosity functions are plotted in Figure 5 .

We adapted the models developed by Blakeslee \& Tonry (1995) to check our estimate of the residual variance contributed by GCs and galaxies. If the GC specific frequency is $S_{N}=7$ for

NGC 4889 (Blakeslee \& Tonry 1995) then $\sigma_{G C}^{2} / \sigma_{S B F}^{2} \approx 0.6$, which corresponds to a correction of $P_{r} \approx 0.4 P_{0}$. We note, however, that the specific frequency measured by Blakeslee \& Tonry is smaller in the inner regions of NGC 4889 by a factor of two to three. The variance is proportional 


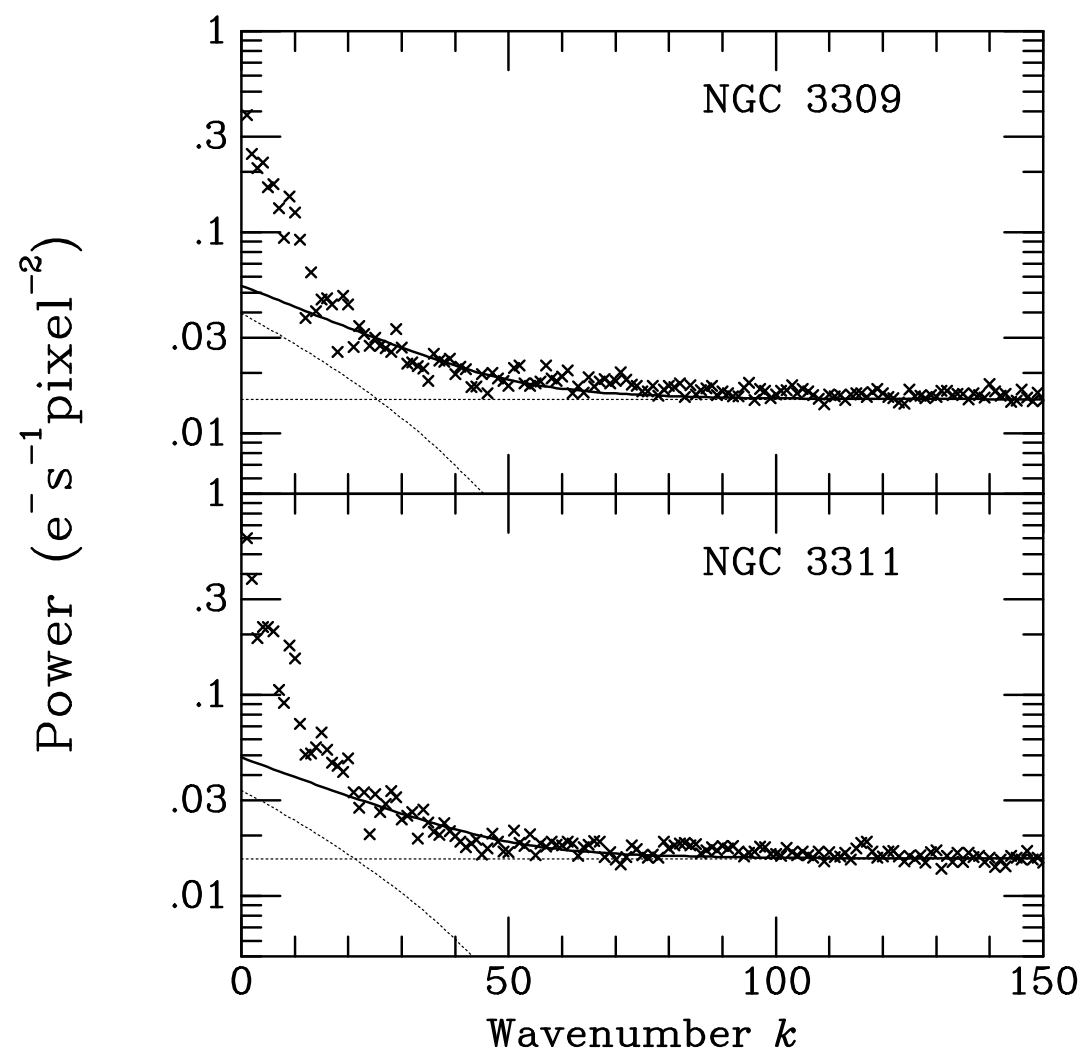

Fig. 3.- Power spectra are plotted for NGC 3309 and NGC 3311 in the Hydra cluster. Solid lines indicate the sum of $P_{0} \times E(k)+P_{1}$; the dotted lines are the individual components. The spectrum shown for NGC 3309 is for all three annuli $\left(2^{\prime \prime}<r<48^{\prime \prime}\right)$, while the spectrum for NGC 3311 excludes the inner annulus. 


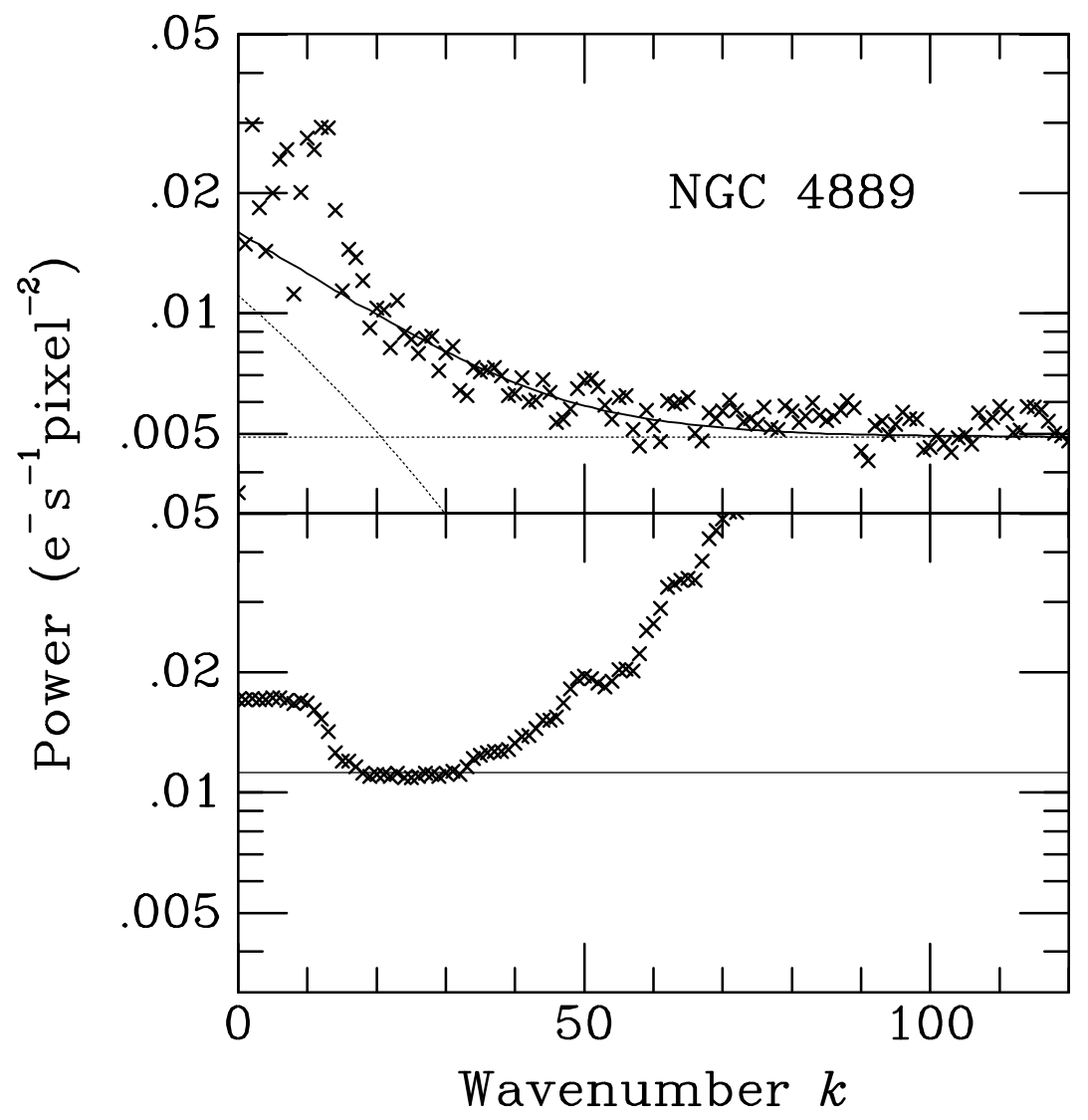

Fig. 4. - The power spectrum for the annular region $12^{\prime \prime}<r<24^{\prime \prime}$ of NGC 4889 is plotted in the top panel. The fitted lines are defined as in Figure 3. The lower panel demonstrates the sensitivity of the $P_{0}$ fit to starting wavenumber (i.e., ignoring wavenumbers lower than $k$ in the fit). We fitted the power spectrum in the range $20<k<33$ and the line in the lower plot indicates the adopted $P_{0}$ value. 


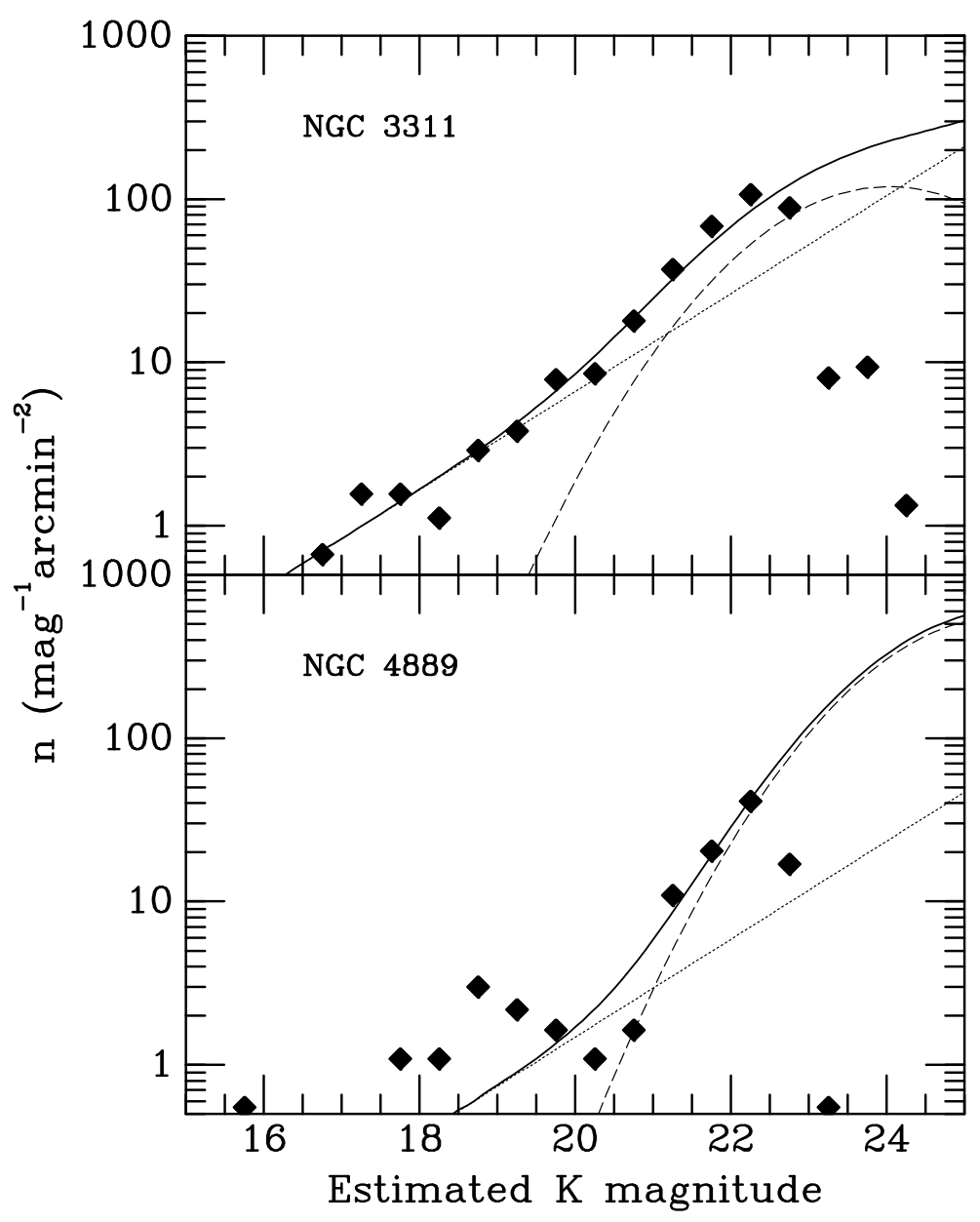

Fig. 5.- Luminosity functions are fitted to photometry of objects measured in the $I$-band image and translated to $K$ by assuming uniform $(I-K)$ colors for GCs and galaxies. The extrapolated luminosity functions are used to compute the variance from GCs and galaxies fainter than the completeness limit, which is $K \approx 22.5$ for these observations. The dotted and dashed lines show the individual fits to the galaxy and GC luminosity functions, respectively, and the solid line is their sum. The GCs in the Hydra image were measured as a single population centered on NGC 3311. 
to $S_{N}$, so we expect to find $P_{r} \approx 0.2 P_{0}$ within $48^{\prime \prime}$ of the center. Examination of the $P_{r} / P_{0}$ values listed in Table 2 confirms that the correction for undetected GCs and galaxies is less than $20 \%$ for NGC 4889. This assumes no additional $P_{g}$ correction for residual spatial variance in the background. If $P_{g}$ is subtracted from $P_{0}$ prior to making the comparison to $P_{r}$, we find that $P_{r}$ is 0.2 to 0.4 times the fluctuation power, just as the models predict.

NGC 3311 in Hydra has a high specific frequency $S_{N}=15$ (McLaughlin et al. 1995), but the Hydra cluster is much closer than Coma and the optical images are comparably deep, so the GCLF for NGC 3311 is better sampled (in fitting the GCLF, we assumed that all GCs detected belong to the NGC 3311 system). The $P_{r}$ correction for the Hydra galaxies is less than $2 \%$ for most annuli. Subtracting $P_{g}$ from $P_{0}$ results in a GC and galaxy contribution to the fluctuation power between 3 and $6 \%$. The model predicts a $P_{r}$ correction of $0.25 P_{0}$ for NGC 3311. It is likely that $S_{N}$ is lower near the center of NGC 3311 where the galaxy is brightest.

It is encouraging how well the point-source masks derived from optical data reduce the residual variance from undetected GCs and galaxies. Even though several assumptions about the GCLF must be made to compute $P_{r}$, the fluctuation magnitude is not sensitive to the exact form of the luminosity function. For example, if the distance used to fix the peak of the GCLF for NGC 4889 is changed from 70 to $110 \mathrm{Mpc}$, the result is a change of only $0.03 \mathrm{mag}$ in $\bar{m}_{K^{\prime}}$. Similarly, changing the width of the GCLF, or the relative fractions of galaxies and GCs, or the bright cutoff magnitude, or any of the other input parameters, had an even smaller effect on the final fluctuation magnitude. These details may be important for measuring a distance based on the absolute magnitude of the GCLF peak, but they do not significantly affect the $K^{\prime}$ fluctuation magnitude. There is sufficient covariance between the fit parameters that if the fit to either the GC or galaxy luminosity function is in error, the fitting procedure will compensate by adjusting the other, resulting in a fit that comes close to correctly predicting $P_{r}$.

\subsection{Additional Sources of Residual Variance}

After correcting for undetected GCs and galaxies, we still found that $P_{0}$ increased significantly with radius as the $S / N$ decreased. Jensen et al. (1996) demonstrated that low- $S / N$ IR SBF observations can be biased by the presence of residual spatial patterns resulting from dark current variations, flat fielding, and subtraction or masking of faint, diffuse objects in the presence of a noisy background. Bright objects leave residual dark current in the HAWAII array that decay with time, adding to the background noise on scales corresponding to the spacing between dithered observations. The noise in our data shows an increase in power at low wavenumbers $(k<50)$ above the fitted constant white noise $P_{1}$ (Figure 6). We estimated that the maximum radial change in $\bar{m}_{K^{\prime}}$ within $50^{\prime \prime}$ of the galaxy center due to hypothetical stellar population variations is $\lesssim 0.5$ mag. The radial gradients in $P_{0}$ on the order of a factor of three must therefore be the result of residual variance that is proportional to the area of the annulus. 
Table 2. $\quad K^{\prime}$ SBF Magnitudes in Hydra and Coma

\begin{tabular}{cccccccc}
\hline \hline Galaxy & Radius (") & $P_{0}\left(\mathrm{e}^{-} \mathrm{s}^{-1} \mathrm{pixel}^{-1}\right)$ & $P_{0} / P_{1}$ & $P_{r} / P_{0}$ & $P_{g} / P_{0}$ & $\xi$ & $\bar{m}_{K^{\prime}}(\mathrm{mag})$ \\
\hline \multirow{2}{*}{ NGC 3309 } & $2-12$ & $1.62 \pm 0.14 \times 10^{-2}$ & 7.2 & 0.00 & 0.29 & 1.65 & $27.72 \pm 0.11$ \\
& $12-24$ & $2.88 \pm 0.10 \times 10^{-2}$ & 3.5 & 0.01 & 0.60 & 0.44 & $27.74 \pm 0.13$ \\
& $24-48$ & $7.42 \pm 0.39 \times 10^{-2}$ & 2.3 & 0.01 & 0.84 & 0.12 & $27.72 \pm 0.42$ \\
& $2-48$ & $3.95 \pm 0.14 \times 10^{-2}$ & 2.7 & 0.01 & 0.72 & 0.25 & $27.78 \pm 0.20$ \\
NGC 3311 & $2-12$ & $1.09 \pm 0.21 \times 10^{-2}$ & 3.1 & 0.04 & 0.42 & 0.74 & $28.42 \pm 0.22$ \\
& $12-24$ & $2.34 \pm 0.10 \times 10^{-2}$ & 2.7 & 0.02 & 0.48 & 0.58 & $27.69 \pm 0.13$ \\
& $24-48$ & $3.91 \pm 0.23 \times 10^{-2}$ & 2.1 & 0.02 & 0.67 & 0.27 & $27.66 \pm 0.27$ \\
& $12-48$ & $3.35 \pm 0.16 \times 10^{-2}$ & 2.2 & 0.02 & 0.64 & 0.31 & $27.72 \pm 0.23$ \\
NGC 4889 & $2-12$ & $0.83 \pm 0.12 \times 10^{-2}$ & 6.0 & 0.15 & 0.19 & 1.90 & $28.55 \pm 0.16$ \\
& $12-24$ & $1.11 \pm 0.02 \times 10^{-2}$ & 2.3 & 0.15 & 0.53 & 0.33 & $29.03 \pm 0.13$ \\
& $24-48$ & $2.53 \pm 0.12 \times 10^{-2}$ & 1.6 & 0.11 & 0.76 & 0.09 & $29.09 \pm 0.33$ \\
& $2-24$ & $0.86 \pm 0.05 \times 10^{-2}$ & 2.9 & 0.18 & 0.41 & 0.55 & $28.99 \pm 0.11$ \\
& $12-48$ & $1.88 \pm 0.08 \times 10^{-2}$ & 1.8 & 0.12 & 0.69 & 0.15 & $29.05 \pm 0.24$ \\
\hline
\end{tabular}




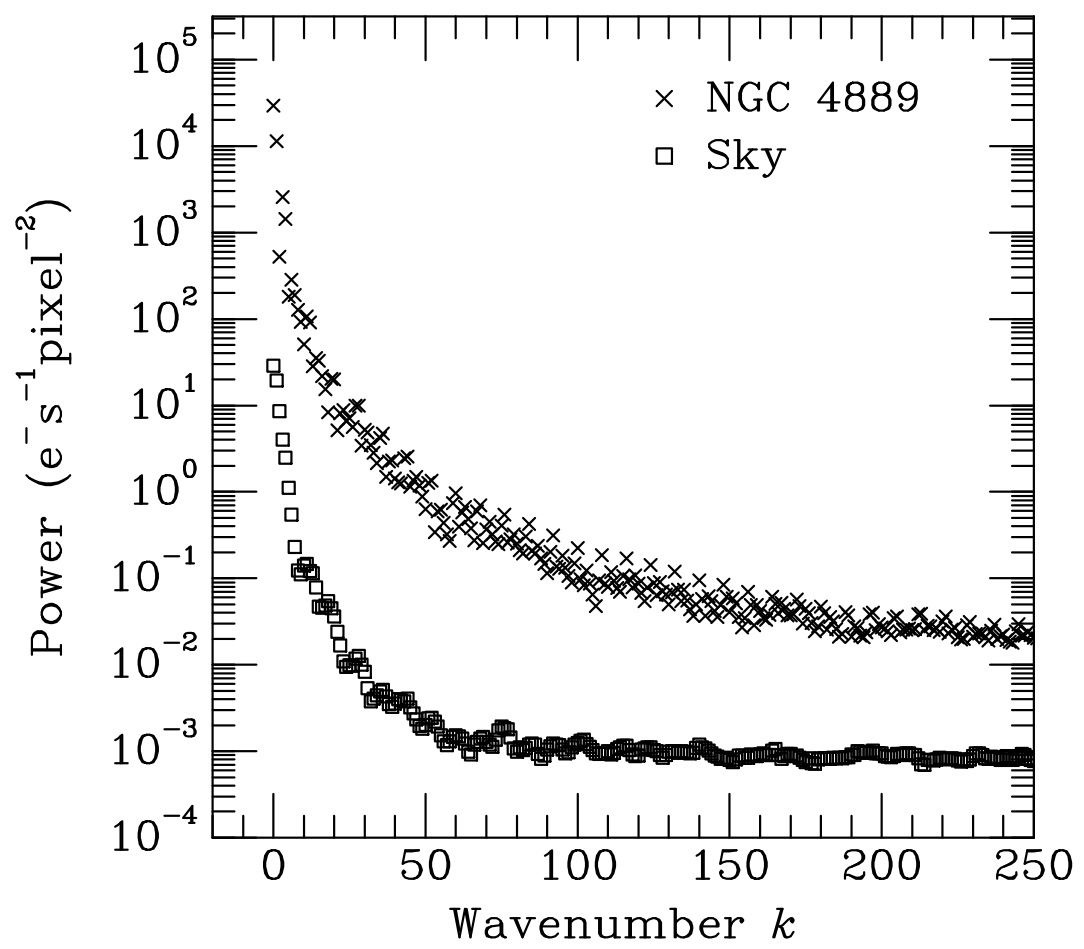

Fig. 6.- The power spectrum for NGC 4889 is shown again, but unscaled by the mean galaxy brightness as in Figure 1 . The figure also shows the power spectrum taken from an adjacent "sky" region approximately $1^{\prime}$ north of the center of NGC 4889. The deviation of the sky spectrum from a flat white-noise power spectrum is evident, particularly for $20<k<50$ where the fits are typically performed. Some of the excess power is from stellar fluctuations in the outer regions of NGC 4889. Subtracting the sky power spectrum from the data spectrum gives the variances listed in Table 3 , which are consistent with the $\bar{m}_{K^{\prime}}$ magnitudes calculated by assuming a uniform residual variance $P_{g}$ proportional to $P_{1}$. The consistency of our final $\bar{m}_{K^{\prime}}$ values between annuli suggests that we are correctly removing the extra power present in the background sky spectrum for $20<k<50$. 
To determine the importance of residual variance in our images, we measured the power spectrum of four relatively empty regions of the Coma image as far from the center of the galaxy as the field of view would permit. GCs and background galaxies were masked as before. The power spectrum for the sky regions do contain some SBF signal from the galaxy, but background noise dominates the stellar fluctuations at these radii. The power spectrum of one of the regions is shown in Figure 6 and is clearly not flat, with increasing power towards $k=0$. Comparison of the power spectra from various regions in the image show that the deviation from a constant noise spectrum $\left(P_{1}\right.$ constant with $\left.k\right)$ is roughly proportional to $P_{1}$. The additional noise in the region of the spectrum where the fits are performed $(20<k<50)$ can be a significant fraction of $P_{0}$.

To remove the background spatial variance, we subtracted the uniform correction $P_{g}$ that best mitigates the radial gradient in $P_{0}$. Because the residual variance is assumed to be uniform across the field of view, $P_{g}$ is proportional to the area of the region being analyzed. $P_{1}$ also increases with the area of the annulus, and $P_{g}$ is roughly proportional to $P_{1}$. Adopting a uniform residual variance correction is a simple way to deal effectively with the power in the spectrum contributed by residual patterns in the image. The levels of $P_{g}$ required to correct for the residual variance in our data were quite large, often $50 \%$ or more of the total power measured. In terms of the sky brightness, the $P_{g}$ corrections were comparable to those measured by JTL: for NGC $3309 P_{g}$ was $0.117 \%$ of the sky brightness; NGC 3311 was best corrected with $P_{g}=0.10 \%$ of the sky level; for NGC 4889 in Coma we applied a correction of $0.07 \%$ of the sky brightness. These corrections are large compared to $P_{0}$ for the distant galaxies in this study, but they are comparable to the corrections applied by JTL that were typically $0.1 \%$ of the background level.

Subtracting a uniform residual variance $P_{g}$ does a reasonably good job of removing the radial gradient in $P_{0}$, but does it correctly remove the noise observed in the power spectrum of the sky region? To answer this question, we took the power spectra measured in the four surrounding regions and subtracted them individually from the data power spectrum for NGC 4889 prior to measuring $P_{0}$. The sky power spectra were measured in apertures with the same radii as those used to measure $P_{0}$ in NGC 4889. The power spectrum from one region is shown in Figure 6,

Table 3. NGC 4889 Fluctuation Amplitudes with Sky Power Spectra Subtracted

\begin{tabular}{|c|c|c|c|c|c|}
\hline \multirow{2}{*}{$\begin{array}{l}\text { Radius } \\
\left({ }^{\prime \prime}\right)\end{array}$} & \multicolumn{4}{|c|}{ Power $\left(\times 10^{-2} \mathrm{e}^{-} \mathrm{s}^{-1}\right.$ pixel $\left.^{-1}\right)$} & \multirow{2}{*}{$\begin{array}{l}\text { Estimated } \\
\text { uncertainty }\end{array}$} \\
\hline & Sky 1 & Sky 2 & Sky 3 & Sky 4 & \\
\hline $2-12$ & 0.48 & 0.60 & 0.51 & 0.29 & 0.09 \\
\hline $12-24$ & $<0$ & 0.49 & 0.43 & $<0$ & 0.13 \\
\hline $2-24$ & 0.20 & 0.54 & 0.45 & $<0$ & 0.13 \\
\hline
\end{tabular}


along with the unscaled power spectrum from NGC 4889 for reference (in Figure 4 the power spectrum was normalized by the mean galaxy brightness). Table 3 lists the fluctuation powers measured in the inner two regions after subtracting the power spectrum from the specified sky region, along with an estimate of the uncertainty for each annulus. The variances listed in Table 3 are consistent with the values determined by applying the $P_{g}$ correction; however, it is clear that variations between power spectra from different regions of the image significantly affect the $\bar{m}_{K^{\prime}}$ measured. In some cases the sky power spectrum had so much power at low wavenumbers that subtracting it from the galaxy power spectrum resulted in a negative variance. We attempted to pick typical areas of the image in which to measure the sky power spectrum. However, if the region near the center of NGC 4889 is as grubby as some of the lower- $S / N$ outer regions of the frame (which it should not be), then NGC 4889 could be more distant than we have estimated by perhaps $\sim 0.5$ mag.

The variances listed in Table 3 confirm that the method of correcting for residual variance by subtracting $P_{g}$ is a reasonable way to account for power in the sky spectrum. While this experiment cannot rule out the possibility that the majority of the power measured is the result of structure in the noise, it constrains the range of fluctuation magnitudes. According to the data listed in Table 3 , $\bar{m}_{K^{\prime}}$ must be brighter than $\sim 29.6$ (corresponding to a power of $0.2 \times 10^{-2} \mathrm{e}^{-} \mathrm{s}^{-1}$ pixel $^{-1}$ ), since sky power spectra that subtracted enough power in the fit region to get this fluctuation magnitude also over-subtracted other annuli. On the other hand, $\bar{m}_{K^{\prime}}$ cannot be any brighter than $\sim 28.5 \mathrm{mag}\left(0.6 \times 10^{-2} \mathrm{e}^{-} \mathrm{s}^{-1}\right.$ pixel $\left.^{-1}\right)$. The fluctuation magnitudes we compute for NGC 4889 by subtracting a uniform background spatial variance $P_{g}$ to minimize the radial change in $P_{0}$ fall comfortably within this range (Table 2). The sky power subtraction experiment was not repeated for the Hydra data because the galaxies almost completely fill the field of view.

Assuming uniform colors for the GC and galaxy populations to determine the $P_{r}$ correction potentially introduces a bias in the fluctuation magnitudes we measure. Some faint galaxies are extremely red, and if they are not masked (because they are fainter than the completeness limit in $I$ ), they will add more flux to the SBF power spectrum at $K^{\prime}$ than we subtract with $P_{r}$. Approximately four objects were easily detected in the $K^{\prime}$ image of NGC 4889 that were below the completeness limit in the $I$-band image. These were masked prior to computing $P_{0}$, but additional faint red galaxies certainly lurk in the noise. Thus the $P_{r}$ correction is probably underestimated. At this point we cannot say how much additional power must be subtracted from $P_{0}$ to account for very red objects in our field without knowing the color distributions of GCs and background galaxies. At an approximate level, though, we account for the deficit in $P_{r}$ with our uniform residual variance correction $P_{g}$. If the extremely red objects (mostly background galaxies) are distributed uniformly in the field of view, then they will most strongly influence the outer annuli, causing a radial increase in $P_{0}$. By adopting a level of residual variance $P_{g}$ that best removes the radial trend in $P_{0}$, we simultaneously correct for both residual patterns and uniformly distributed red galaxies. We suggest that in future observations, optical integrations be deep enough to reduce 
the $P_{r}$ correction to negligible levels, thereby reducing the systematic error associated with $P_{r}$. For this paper, we increased the estimated uncertainty for $P_{r}$ listed in Table 4 to account for the potential bias. We are confident that the bias is largely removed by our residual variance subtraction.

\subsection{Reliability of the SBF Measurements}

The stellar SBF $S / N$ (with residual variances subtracted) was computed for each annulus using the definition from JTL:

$$
\xi=\frac{P_{0}-P_{r}-P_{g}}{P_{1}+P_{g}}
$$

and listed in Table 2. In calibrating the $K^{\prime}$ SBF scale, JTL showed that measurements with $\xi<1$ are unreliable. Only the innermost regions of the galaxies in the current study have strong enough signals to meet this criterion. Smaller annuli are also the least sensitive to the residual variance correction. After the residual variance bias has been removed, we find that the fluctuation magnitudes are quite consistent, in spite of the low $S / N$ in the outer apertures.

Fluctuation magnitudes measured in the innermost annulus $\left(2^{\prime \prime}-12^{\prime \prime}\right)$ of NGC 3311 and NGC 4889 fail to match the outer annuli. The inner region of NGC 3311 has an anomalously faint

$\bar{m}_{K^{\prime}}$ magnitude. The center of this galaxy has an unusual off-center nucleus, and the galaxy fitting routine fails near the center. We masked the center of NGC 3311 prior to measuring the SBFs, but it is not clear if the anomalous fluctuation magnitude is the result of a distinct stellar population in the nuclear region of NGC 3311 or due to a failure in the SBF analysis. It is also possible that the nuclear region of NGC 3311 is obscured by dust. A uniform distribution of dust will reduce the measured fluctuation amplitude. The inner annulus is excluded from the power spectrum plotted in Figure 3 and from the calculation of $\bar{m}_{K^{\prime}}$ for this galaxy. SBFs in the central region of NGC 4889 are brighter than in the outer annuli after correction for residual variance. Both $I$ and $K^{\prime}$ images show a similar pattern near the center that may be blended images of GCs or stellar SBFs. They may also signal the failure of our galaxy model fitting routine, which sometimes has difficulty very near the centers of galaxies. Since the fluctuation magnitude is 0.5 mag brighter in the inner annulus than in any of the other annuli (including composite regions that include the inner annulus but weight it less heavily), it seems likely that the inner measurement is biased by point sources or a deviation of our model from the real surface brightness profile near the center. The power spectrum shown in Figure 4 is for the second annulus $\left(12^{\prime \prime}<r<24^{\prime \prime}\right)$.

Uncertainties in many different input parameters affect the fluctuation magnitudes measured. To estimate the uncertainty in $\bar{m}_{K^{\prime}}$, we changed the input values for each parameter in turn and measured the change in $\bar{m}_{K^{\prime}}$. The uncertainties from PSF fitting, galaxy subtraction, residual background subtraction, correction for GCs, and so forth were added in quadrature. This procedure assumes that the uncertainties are independent, which is not strictly true; significant covariances exist between sky level subtraction and galaxy profile fitting, for example, or between 
the photometric zero point and the PSF normalization. The individual uncertainties for the Hydra and Coma observations are specified in detail in Table 1 . Some of these uncertainties are systematic to our study, so the uncertainties in our final fluctuation magnitudes are larger than the scatter between individual measurements. The uncertainty in the photometric zero point, for example, is the same for both galaxies in the Hydra image. The uncertainties in fluctuation magnitudes measured in different annuli have a large covariance as well.

Other sources of uncertainty could be either random or systematic, or both. The estimated uncertainty in the correction for any residual spatial variance $\left(P_{g}\right)$ is the dominant source of uncertainty listed in Table 4. Whether or not this uncertainty affects all annuli equally (systematically) or not depends on the distribution of the background variance in the image. The uncertainty in $P_{g}$ is particularly difficult to estimate because many sources of variance add to the power measured (residual dark current from saturated sources, unmasked objects in sky images, etc.). We corrected for background spatial variance as well as possible and estimated realistic uncertainties, but adding systematic uncertainties in quadrature leads to an underestimate of the uncertainty in $\bar{m}_{K^{\prime}}$. To account for the fact that the uncertainty in $P_{g}$ has both random and systematic components, we estimated an additional systematic uncertainty from residual background variance subtraction and included it in Table 4. The correction for residual variance from undetected GCs and galaxies also has random and systematic uncertainties associated with it, and we added an additional systematic uncertainty in $P_{r}$ to Table 4 . The estimated systematic uncertainties were not included in the $\bar{m}_{K^{\prime}}$ uncertainties, but were added in quadrature to compute the uncertainties in the distance moduli, distances, and Hubble constant.

Although it is difficult to understand all the possible covariances in the estimated uncertainties, we feel that we have fairly estimated the uncertainty in our measurement of $\bar{m}_{K^{\prime}}$. The uncertainties reported by JTL were determined by adding the individual uncertainties in quadrature, and the fits to that data had $\chi^{2}$ values per degree of freedom between 0.5 and 2 , suggesting that the estimated uncertainties were reasonable. In the final analysis, our fluctuation magnitudes are consistent, indicating that residual variance has been removed properly in annuli of different sizes.

\subsection{Distances}

To compute the distance moduli, we used the $K^{\prime}$ SBF calibration from JTL based on Cepheid distances to M31 and the Virgo cluster. JTL found that $\bar{M}_{K^{\prime}}=-5.61 \pm 0.12$, in excellent agreement with Jensen et al. (1996), Luppino \& Tonry (1993), and Pahre \& Mould (1994). No significant trend in $\bar{M}_{K^{\prime}}$ was found as a function of galaxy color for the limited range $1.15<(V-I)<1.27$. There are no published $(V-I)$ colors for the galaxies in this sample. To confirm that the calibration applies to the Coma and Hydra cluster galaxies, we estimated $(V-I)$ colors using published $(V-K)$ values from (Persson, Frogel, \& Aaronson 1979) and mean $(I-K)$ colors for our calibration galaxies (JTL). For the Virgo, Fornax, and Eridanus galaxies with $(V-I)>1.2$, the mean $(I-K)=2.2$, implying $(V-I) \approx 1.23$ for NGC 4889 . The estimated 
Table 4. SBF Uncertainties for Hydra and Coma

\begin{tabular}{ccc}
\hline \hline $\begin{array}{c}\text { Source of } \\
\text { Uncertainty }\end{array}$ & $\begin{array}{c}\text { Hydra } \\
\sigma(\mathrm{mag})\end{array}$ & $\begin{array}{c}\text { Coma } \\
\sigma(\mathrm{mag})\end{array}$ \\
\hline $\begin{array}{c}\text { Random Uncertainties } \\
\text { Photometry .............. }\end{array}$ & 0.01 & 0.01 \\
PSF normalization......... & 0.02 & 0.03 \\
$P_{0}$ fit (typical) ............. & 0.04 & 0.05 \\
Residual sky and & & \\
model galaxy subtraction & 0.05 & 0.03 \\
GCs and galaxies ........... & 0.03 & 0.05 \\
Residual noise subtraction .. & $0.03-0.41$ & $0.02-0.32$ \\
& & \\
Systematic Uncertainties & & \\
GCs and galaxies ........... & 0.05 & 0.10 \\
Residual noise subtraction .. & 0.16 & 0.15 \\
$K^{\prime}$ SBF Calibration......... & 0.06 & 0.06 \\
Cepheid zero point.......... & 0.10 & 0.10 \\
\hline
\end{tabular}


$(V-I)$ color for NGC 4889 is typical of the JTL sample, and we adopt the calibration from JTL without modification. The $(V-I)$ colors were estimated to be 1.25 and 1.31 for NGC 3309 and NGC 3311, respectively. NGC 3311 may be redder than the galaxies used by JTL to calibrate the $K^{\prime} \mathrm{SBF}$ distance scale, but the uncertainty on the estimated $(V-I)$ color is $\sim 0.1 \mathrm{mag}$ so we cannot conclude that NGC 3311 is different from the galaxies in the JTL sample. The same calibration of $\bar{M}_{K^{\prime}}$ was used for the Hydra galaxies. Because the $\mathrm{K}$ corrections predicted by old stellar population models are negligible at $K$ (JTL), we computed the luminosity distance (in Mpc) using the relation

$$
d_{L}=10^{0.2(\bar{m}-\bar{M})-5}
$$

and found a distance of $84.7 \pm 9.8 \mathrm{Mpc}$ to NGC 4889. The distances to the Hydra cluster galaxies were computed in the same way giving distances of $46.3 \pm 4.9$ and $45.7 \pm 5.0 \mathrm{Mpc}$ to NGC 3309 and NGC 3311. The distances are remarkably consistent (the uncertainties for NGC 3309 and NGC 3311 are correlated because both measurements were made in the same image and share many of the same uncertainties). Fluctuation magnitudes, distance moduli and distances are listed in Table 5. The distance uncertainties include the systematic uncertainties from the calibration, added in quadrature.

The astrometric satellite Hipparcos has recently provided important parallax distances to Cepheid variable stars in the Galaxy. Hipparcos measurements suggest that the Cepheid distance scale should be increased by $\sim 10 \%$ (Feast \& Catchpole 1997). If confirmed, the Hipparcos results may require us to modify the Cepheid calibration of IR SBFs. Madore \& Freedman (1997), however, find that the change in Cepheid zero point suggested by the Hipparcos data is smaller than the uncertainty in the Cepheid calibration due to uncertainties in metallicity or reddening corrections, so it is premature to adopt a new calibration at this point.

\section{The Hubble Constant}

We computed the Hubble constant using the equation

$$
H_{0}=\frac{c z}{d_{L}} .
$$

At the distance of the Coma cluster $(z=0.025)$, the cosmological correction to the luminosity distance $d_{L}$ is less than $1 \%$ for $q_{0}=0.5$ (Kolb \& Turner 1990, Eq. 2.52). We used the distances and velocities listed in Table 5 to compute the tabulated values of $H_{0}$. Mean cluster velocities in the CMB frame were drawn from Han \& Mould (1992). The cluster velocities reported by Faber et al. (1989) are virtually identical $\left(4033 \mathrm{~km} \mathrm{~s}^{-1}\right.$ for Hydra and $7202 \mathrm{~km} \mathrm{~s}^{-1}$ for Coma). Values of the Hubble constant for the Hydra and Coma clusters are consistent (Table 5), giving a mean Hubble constant of $H_{0}=87 \pm 11 \mathrm{~km} \mathrm{~s}^{-1} \mathrm{Mpc}^{-1}$. The uncertainties for the three measurements are not completely independent because they are drawn from the same observations.

The IR SBF Hubble constant measured in this study is consistent with an extensive optical 
( $I$-band) SBF survey of several hundred galaxies that gives $H_{0}=81 \pm 6 \mathrm{~km} \mathrm{~s}^{-1} \mathrm{Mpc}^{-1}$ (Tonry et al. 1997). A detailed comparison of the $I$-band SBF distance scale with several other distance estimators is included in Tonry et al. (1997). Lauer et al. (1997) and Ajhar et al. (1997) used the Hubble Space Telescope (HST) to measure $I$-band SBF distances to several Abell clusters. Calibrating the brightest cluster galaxy distance scale using $I$-band SBFs gives $H_{0}=89 \pm 10$ $\mathrm{km} \mathrm{s}^{-1} \mathrm{Mpc}^{-1}$.

The distance we measured to NGC 4889 is shorter than the distance of $102 \pm 14 \mathrm{Mpc}$ recently measured by Thomsen et al. (1997) using the HST to probe $I$-band SBFs in the Coma cluster galaxy NGC 4881. The two distances agree at the 1- $\sigma$ level; nevertheless, we do not know if the difference between our measurement and theirs is significant. Both our measurement and that of Thomsen et al. have low $S / N$ (Thomsen et al. accumulated only 0.7 SBF photons per noise photon). The detectors, filters, and procedures used by Thomsen et al. are significantly different from those used for this study, making it difficult to compare possible sources of systematic error. Previous IR SBF studies have found a few galaxies with anomalously bright fluctuation magnitudes (Luppino \& Tonry 1993; Pahre \& Mould 1994; Jensen et al. 1996; JTL). Some were shown to be anomalous because of observational bias at low $S / N$ (JTL); others may have unusual stellar populations and await follow-up observations to confirm their bright fluctuation magnitudes. It is possible that NGC 4889 in Coma is a galaxy with anomalously bright IR SBFs because it possesses an unusual stellar population. On the other hand, there is no obvious reason why NGC 4889 should have a different stellar population from the other galaxies we have studied, and we believe our distance measurement to be reliable. Indeed, we have measured IR SBFs in three galaxies in two clusters with remarkably consistent results (although the agreement between NGC 3309 and NGC 3311 is partially a result of the fact that they were observed simultaneously).

These are the first IR SBF measurements to be made at such large distances, and additional high- $S / N$ observations will be needed to reduce the uncertainty in the SBF distance to Coma. The HST with NICMOS and new large telescopes equipped with large-format IR detectors will allow us to confirm our results using larger samples. Improvements in observational and data reduction techniques will reduce residual variances and increase the reliability of IR SBF measurements. In future ground-based observations we will use deep optical images to identify faint objects in sky fields as we did in the galaxy images to improve sky subtraction and reduce background patterns from unmasked objects in the sky fields. The influence of residual patterns from bright saturated objects will be reduced by using shorter exposure times and by dithering in only one direction. By dithering along one axis only, the power from residual patterns will be confined to a line and can be masked prior to fitting the power spectrum. These modifications to the observing techniques should significantly reduce the amplitude of residual background patterns that can bias low- $S / N$ IR SBF measurements. 


\section{Summary}

This paper describes our attempt to extend the IR SBF distance scale to the Coma cluster. We measured $K^{\prime}$ SBFs in two Hydra cluster giant elliptical galaxies and in NGC 4889 in Coma. Our conclusions are summarized as follows:

1. IR SBF distance measurements to three galaxies in the Hydra and Coma clusters imply a Hubble constant $H_{0}=87 \pm 11 \mathrm{~km} \mathrm{~s}^{-1} \mathrm{Mpc}^{-1}$ if sources of residual variance have been subtracted correctly. Results from both clusters are consistent. These measurements support the conclusion that Hydra's radial peculiar velocity is small.

2. Fluctuations from globular clusters and background galaxies can be adequately removed, even at distances of $7000 \mathrm{~km} \mathrm{~s}^{-1}$. Modest optical exposures can be used to identify and mask GCs and galaxies in IR images, potentially reducing the variance from undetected GCs and galaxies to negligible levels. Without using optical images, considerably longer IR integrations could be required to reduce the GC correction to similarly low levels.

3. Corrections to remove residual spatial variance in the background are necessary to measure radially consistent fluctuation magnitudes. The power spectrum of the noise in our near-IR images is not independent of wavenumber. For the relatively short integration on NGC 4889 described in this study, the size of the correction for residual variance is large compared to the SBF signal. To achieve reliable results in the future, IR SBF researchers should strive to produce images that are as clean and flat as possible, and to integrate long enough under good seeing conditions to achieve a high $S / N$. Additional improvements in observing techniques may also help reduce residual background variance in future IR SBF measurements.

The SBF observations described in this study were obtained using relatively short integration times on a modest $2.24 \mathrm{~m}$ telescope and using the first of a new generation of $1024^{2}$-pixel IR detectors. Future IR SBF studies of galaxies to $100 \mathrm{Mpc}$ and beyond will benefit from NICMOS on the Hubble Space Telescope with its low background, and from the new generation of $8 \mathrm{~m}$ class telescopes with clean, large-format IR detectors. For example, the Gemini telescope on Mauna Kea with the NIRI infrared imager under good seeing conditions will make it possible to make high-S/N K-band SBF measurements at the distance of the Coma cluster in $\$ 2000$ s per galaxy. With telescopes like Gemini we will be able to reliably measure SBF distances to many galaxies in a distant cluster in a night, making it possible to measure the Hubble constant, map peculiar velocities, and measure bulk motions on scales much larger than currently possible using SBFs.

We are indebted to John Blakeslee and Alan Dressler who provided deep optical images of NGC 3309, NGC 3311, and NGC 4889. Their images were invaluable in reducing the GC and galaxy contributions to the SBF signal to manageable levels. This research was supported in part by grants STScI GO-06579.01-95A and NSF AST9401519. 
Table 5. $\quad K^{\prime}$ SBF Distances to Hydra and Coma

\begin{tabular}{cccccc}
\hline \hline Galaxy & $\begin{array}{c}\bar{m}_{K^{\prime}} \\
(\mathrm{mag})\end{array}$ & $\begin{array}{c}(\bar{m}-\bar{M})_{K^{\prime}} \\
(\mathrm{mag})\end{array}$ & $\begin{array}{c}d \\
(\mathrm{Mpc})\end{array}$ & $\begin{array}{c}v^{\mathrm{a}} \\
\left(\mathrm{km} \mathrm{s}^{-1}\right)\end{array}$ & $\begin{array}{c}H_{0} \\
\left(\mathrm{~km} \mathrm{~s}^{-1} \mathrm{Mpc}^{-1}\right)\end{array}$ \\
\hline NGC 3309 & $27.72 \pm 0.11$ & $33.33 \pm 0.23$ & $46.3 \pm 4.9$ & $4054 \pm 296$ & $87 \pm 11$ \\
NGC 3311 & $27.69 \pm 0.13$ & $33.30 \pm 0.24$ & $45.7 \pm 5.0$ & $4054 \pm 296$ & $89 \pm 12$ \\
NGC 4889 & $29.03 \pm 0.13$ & $34.64 \pm 0.25$ & $84.7 \pm 9.8$ & $7186 \pm 428$ & $85 \pm 11$ \\
\hline
\end{tabular}

${ }^{\mathrm{a}}$ Han \& Mould 1992 


\section{REFERENCES}

Ajhar, E. A., Lauer, T. R., Tonry, J. L., Blakeslee, J. P., Dressler, A., Holtzman, J. A., \& Postman, M. 1997, AJ, 114, 626

Ajhar, E. A. \& Tonry, J. L. 1994, ApJ, 429, 557

Blakeslee, J. P. \& Tonry, J. L. 1995, ApJ, 442, 579

Burstein, D. \& Heiles, C. 1984, ApJS, 54, 33

Casali, M. \& Hawarden, T. G. 1992, JCMT-UKIRT Newsletter, 4, 33

Cohen, J. G., Frogel J. A., Persson, S. E., \& Elias, J. H. 1981, ApJ, 249, 481

Faber, S. M., Wegner, G., Burstein, D., Davies, R. L., Dressler, A., Lynden-Bell, D., \& Terlevich, R. J. 1989, ApJS, 69, 763

Feast, M. W. \& Catchpole, R. M. 1997, MNRAS, 286, L1

Frogel, J. A., Persson, S. E., Aaronson, M., \& Matthews, K. 1978, ApJ, 220, 75

Han, M. \& Mould, J. R. 1992, ApJ, 396, 453

Hodapp, K.-W. et al. 1996, New Astronomy, 1, 177

Jacoby, G. H., Branch, D., Ciardullo, R., Davies, R. L., Harris, W. E., Pierce, M. J., Pritchet, C. J., Tonry, J. L., \& Welch, D. L. 1992, PASP, 104, 599

Jensen, J. B., Luppino, G. A., \& Tonry, J. L. 1996, ApJ, 468, 519

Jensen, J. B., Tonry, J. L., \& Luppino, G. A. 1998, ApJ, in press (JTL)

Kolb, E. W. \& Turner, M. S. 1990, in The Early Universe (Redwood City: Addison Wesley), 42

Lauer, T. \& Postman, M. 1994, ApJ, 425, 418

Lauer, T. R., Tonry, J. L., Postman, M., Ajhar, E. A., \& Holtzman, J. A. 1997, ApJ, in press

Livio, M., Donahue, M., \& Panagia, N. 1997, The Extragalactic Distance Scale (Cambridge: Cambridge University Press)

Luppino, G. A. \& Tonry, J. L. 1993, ApJ, 410, 81

Lynden-Bell, D., Faber, S. M., Burstein, D., Davies, R. L., Dressler, A., Terlevich, R. J., \& Wegner, G. 1988, ApJ, 326, 19

Madore, B. F. \& Freedman, W. L. 1997, ApJ, 492, 110

Mathewson, D. S., Ford, V. L., \& Buchhorn, M. 1992, ApJ, 389, 5 
McLaughlin, D. E., Secker, J., Harris, W. E., \& Geisler, D. 1995, AJ, 109, 1033

Mould, J. R., Staveley-Smith, L., Schommer, R. A., Bothun, G. D., Hall, P. J., Han, M. S., Huchra, J. P., Roth, J., Walsh, W., \& Wright, A. E. 1991, ApJ, 383, 467

Pahre, M. A. \& Mould, J. R. 1994, ApJ, 433, 567

Persson, S. E., Frogel, J. A., \& Aaronson, M. 1979, ApJS, 39, 61

Thomsen, B., Baum, W. A., Hammergren, M., \& Worthey, G. 1997, ApJ, 483, 37

Tonry, J. L., Blakeslee, J. P., Ajhar, E. A., \& Dressler, A. 1997, ApJ, 475, 399

Tonry, J. L. \& Schneider, D. P. 1988, AJ, 96, 807

Wainscoat, R. \& Cowie, L. 1992, AJ, 101, 332

Worthey, G. 1994, ApJS, 95, 107

This preprint was prepared with the AAS LATEX macros v4.0. 
This figure "fig1.jpg" is available in "jpg" format from: http://arxiv.org/ps/astro-ph/9807326v1 
This figure "fig2.jpg" is available in "jpg" format from: http://arxiv.org/ps/astro-ph/9807326v1 